\title{
Biossegurança em procedimentos cirúrgicos odontológicos frente a pandemia do COVID-19
}

\author{
Biosafety in dental surgical procedures in front of the COVID-19 pandemic \\ Bioseguridad en procedimientos quirúrgicos dentales frente a la pandemia COVID-19
}

Recebido: 21/12/2020 | Revisado: 23/12/2020 | Aceito: 28/12/2020 | Publicado: 03/01/2021

\author{
Jefferson Alves Freitas \\ ORCID: https://orcid.org/0000-0002-6357-6301 \\ Faculdade Uninassau Aliança, Brasil \\ E-mail: bomdiadoto@gmail.com \\ Itana Silva Carvalho \\ ORCID: https://orcid.org/ 0000-0003-2332-3331 \\ Faculdade Uninassau Aliança, Brasil \\ E-mail: itanacarvalho1@gmail.com \\ Fernanda Beraldo Alves \\ ORCID: https://orcid.org/ 0000-0002-1792-2517 \\ Faculdade Uninassau Aliança, Brasil \\ E-mail: nanda.balves@gmail.com \\ Natália da Silva Costa \\ ORCID: https://orcid.org/ 0000-0001-5422-8471 \\ Faculdade Uninassau Aliança, Brasil \\ E-mail: nataliacosta_phn@hotmail.com \\ Guereth Alexsanderson Oliveira Carvalho \\ ORCID: https://orcid.org/0000-0003-3286-2943 \\ Universidade Federal do Piauí, Brasil \\ E-mail: guerethcarvalho@gmail.com
}

\begin{abstract}
Resumo
As medidas de biossegurança se intensificaram após o início da pandemia do COVID-19, que é uma doença que pode desenvolver um quadro clínico que varia de infecções assintomáticas a quadros respiratórios graves. Deste modo, cirurgiões-dentistas, assim como outros profissionais da saúde, tiveram que se adequar a critérios mais rigorosos de paramentação. O objetivo desse estudo é apresentar as principais recomendações no que tange a biossegurança nos procedimentos cirúrgicos odontológicos frente a pandemia. Este trabalho é uma revisão sistematizada da literatura, realizada através de artigos publicados na língua inglesa, espanhola e portuguesa, entre 2010 e 2020, publicados na base de dados PUBMED, BVS, RESEARCH GATE, SCIELO, tendo por descritores: odontologia, cirurgia oral, biossegurança, covid-19. Constatou-se que a especialidade de Cirurgia e Traumatologia Bucomaxilofacial é a mais exposta aos riscos de infeções por bioaerossóis, e devido a isto, é necessário o uso de máscaras cirúrgicas. PFF2/N-95, protetores faciais, óculos de proteção e capote descartável, assim como a adoção de protocolos rígidos de higienização, desinfecção, esterilização, e organização do ambiente cirúrgico, ao passo que a Teleodontologia se mostrou como um instrumento auxiliar viável neste período a fim de reduzir os riscos à exposição pelo SARS-CoV-2. O profissional que atua nos atendimentos cirúrgicos de urgência e emergência devem seguir as medidas de biossegurança emitidas pelos órgãos de vigilância em saúde a fim de que a saúde do trabalhador seja assegurada, igualmente a do paciente.
\end{abstract}

Palavras-chave: Odontologia; Cirurgia oral; Biossegurança; COVID-19.

\begin{abstract}
Biosafety measures intensified after the beginning of the COVID-19 pandemic, which is a disease that can develop a clinical picture ranging from asymptomatic infections to severe respiratory conditions. Thus, dental surgeons, as well as other health professionals, had to adapt to stricter criteria for dressing. The objective of this study is to present the main recommendations regarding biosafety in dental surgical procedures in the face of the pandemic. This work is a systematic review of the literature, carried out through articles published in English, Spanish and Portuguese, between 2010 and 2020, published in the PUBMED, BVS, RESEARCH GATE, SCIELO databases, with the following descriptors: oral surgery, biosafety, COVID-19. It was found that the specialty of Buccomaxillofacial Surgery and Traumatology is the most exposed to the risks of infections by bioaerosols, and due to this, the use of surgical masks is necessary, PFF2 / N-95, faceshields, goggles and disposable cloak, as well as the adoption of strict protocols for hygiene, disinfection, sterilization, and organization of the surgical environment, whereas Teledentistry proved to be a viable auxiliary instrument in this period in order to reduce the risks to exposure by SARS-CoV-2. The professional
\end{abstract}


who works in urgent and emergency surgical care must follow the biosafety measures issued by the health surveillance agencies in order to ensure that the worker's health is guaranteed, as well as that of the patient.

Keywords: Dentistry; Oral surgery; Biosafety; COVID-19.

\begin{abstract}
Resumen
Las medidas de bioseguridad se intensificaron tras el inicio de la pandemia COVID-19, que es una enfermedad que puede desarrollar un cuadro clínico que va desde infecciones asintomáticas hasta afecciones respiratorias graves. Así, los cirujanos dentistas, así como otros profesionales de la salud, tuvieron que adaptarse a criterios más estrictos de vestimenta. El objetivo de este estudio es presentar las principales recomendaciones en materia de bioseguridad en los procedimientos quirúrgicos dentales ante la pandemia. Este trabajo es una revisión sistemática de la literatura, realizada a través de artículos publicados en inglés, español y portugués, entre 2010 y 2020, publicados en la base de datos PUBMED, BVS, RESEARCH GATE, SCIELO, con los siguientes descriptores: cirugía oral, bioseguridad, COVID-19. Se encontró que la especialidad de Cirugía y Traumatología Buccomaxilofacial es la más expuesta a los riesgos de infecciones por bioaerosoles, por lo que es necesario el uso de mascarillas quirúrgicas. PFF2 / N-95, protectores faciales, goggles y manto desechable, así como la adopción de estrictos protocolos de higiene, desinfección, esterilización y organización del ambiente quirúrgico, mientras que la Teleodontología demostró ser un instrumento auxiliar viable en este período. para reducir los riesgos de exposición al SARS-CoV-2. El profesional que trabaja en la atención quirúrgica de urgencia y emergencia debe seguir las medidas de bioseguridad emitidas por los organismos de vigilancia de la salud a fin de asegurar que la salud del trabajador, así como la del paciente, esté garantizada.
\end{abstract}

Palabras clave: Odontología; Cirugía bucal. Bioseguridad. COVID-19.

\title{
1. Introdução
}

A Odontologia ao longo dos anos tem passado por um notável processo evolutivo, tanto ao que é pertinente à sua área de atuação que é regulamentada pela Lei n 5081 , de 24 de agosto de 1966, a qual define o exercício da profissão e assegura a legitimidade profissional do odontólogo na região bucomaxilofacial, quanto pelos desafios que a comunidade global tem passado, como o impacto da pandemia do COVID-19 que tem imposto a necessidade de novos protocolos de biossegurança à fim de que a saúde de todos os envolvidos na dinâmica do atendimento possam ser preservadas.

Cerca de 1,3 milhões óbitos já foram registrados (Organização Mundial de Saúde [OMS], 2020) e com isto, os cirurgiões-dentistas devem se policiar quanto aos aspectos de risco que a prática laboral pode desempenhar, tendo em vista a possibilidade de infecções principalmente nos procedimentos de urgência, como os cirúrgicos bucomaxilofaciais, que foram os primeiros a serem liberados para o atendimento de urgência e emergência após o início da quarentena.

Teixeira e Valle (2010, p.19), evidenciaram que a biossegurança se caracteriza como o conjunto de ações que visam a prevenção, redução ou eliminação de fatores de risco que podem estar associados à prática de atividades em prol do desenvolvimento técnico-cientifico em detrimento à saúde humana e ao meio ambiente. E partindo disto, é dever do profissional da saúde, assim como da população de modo geral seguir as recomendações dos órgãos de vigilância sanitária, para que as consequências da pandemia do COVID-19 possam ser amenizadas.

O objetivo deste trabalho é apresentar as principais orientações quanto aos procedimentos de biossegurança no atendimento odontológico bucomaxilofacial frente aos riscos de infecção pelo SARCov-2 na saúde humana.

\section{Metodologia}

Este trabalho consiste numa revisão sistematizada da literatura, a qual foi desenvolvida de acordo com as etapas construtivas do protocolo de pesquisa: formulação da questão norteadora "Quais os equipamentos de proteção individual devem ser utilizados no atendimento cirúrgico bucomaxilofacial a fim de reduzir os riscos da infecção pelo SARS-CoV-2?”; busca, seleção e revisão dos estudos, os quais foram publicados em 2020, exceto um artigo do ano de 2010 que salvo seu referencial teórico foi incluído, sendo estes nas línguas inglesa, espanhola e portuguesa, indexados nas bases de dados PUBMED, BVS, RESEARCH GATE, SCIELO, tendo por descritores: cirurgia oral, biossegurança, covid-19. 
A análise crítica de cada artigo foi realizada aos pares, sendo realizado no mínimo por dois pesquisadores simultaneamente, onde os critérios de inclusão foram artigos que abordassem as medidas de biossegurança na prática odontológica em contexto de pandemia, contendo recomendações comportamentais e de manejo, uso de equipamento de proteção individual, e protocolos de triagem à distância para o atendimento de caráter emergencial. De modo que os critérios de exclusão foram artigos os quais não contemplavam o objetivo deste estudo ou que não delimitavam a questão norteadora.

\section{Resultados e discussão}

\subsection{SARS-CoV-2 e COVID-19}

De acordo com a OMS (2020), em escala global, cerca de 66,9 milhões de pessoas já foram infectadas pelo SARSCoV-2, onde mais de 6,6 milhões destes casos foram diagnosticados no Brasil, tendo 176,9 mil óbitos registrados, o que reflete a importância da observância às medidas de biossegurança tanto na prática profissional, quanto na dinâmica dos indivíduos na sociedade.

Freitas, Cavalcante \& Lopes (2020, p.166), definiram a COVID-19 como a doença advinda de uma mutação do coronavírus, o SARS-CoV-2, que pode desenvolver um quadro clínico que varia de infecções assintomáticas a quadros respiratórios graves. Todavia, é válido evidenciar também que após o aumento no número de casos de pneumonia na China, cuja etiologia era desconhecida até o final de 2019, e que apesar das inúmeras tentativas para mantê-la em caráter epidêmico, se disseminou no planeta celeremente, o que fez com que a Organização Mundial de Saúde declarasse como pandemia em março de 2020 (Ludwig \& Zarbock, 2020, p.1).

Xu et.al (2020, p.1), relatou que a angiotensina 2 (ACE2) é expressiva na mucosa bucal, onde é abundante nas células epiteliais da língua, o que evidenciou a suscetibilidade da cavidade oral para a infecção pelo Sars-CoV-2, o que também foi relatado por Li, Zhou, Yang e You (2020, p.1), e partindo disto, houve o alerta à classe odontológica para a elaboração de estratégias preventivas na prática clínica, bem como aos novos comportamentos para o convívio social.

A Agência Nacional de Vigilância Sanitária (ANVISA) alertou através da nota técnica GVIMS/GGTES/ANVISA N ${ }^{\circ}$ 04/2020 que após mais de um semestre de pandemia são verificados casos heterogêneos no Brasil, no que se trata a incidência das infecções pelo SARS-CoV-2, todavia o aumento de casos em outros países tem sugerido a possibilidade de uma segunda onda, e isso revela a necessidade de intensificar as medidas nacionais de prevenção e controle de novos casos.

\subsection{Biosssegurança}

Apesar dos métodos de prevenção a infecções clinico-hospitalares terem sido difundidos, de forma jamais vista, com a eclosão da pandemia do COVID-19, alguns destes cuidados já eram realizados de modo pontual por alguns profissionais e instituições. Sobretudo Segata (2020, p.283), esclareceu que a construção do campo de biossegurança é articulada por áreas distintas do conhecimento, tais como: a ecologia, a epidemiologia, a biotecnologia, a bioética e as humanidades. Sendo que através de uma análise antropológica, ressaltou também que apesar da origem multidisciplinar da biossegurança, há quem acredite que se caracterize apenas como uma especialidade dedicada às ações de contenção de riscos que envolvem à exposição a agentes biológicos com potencial de contaminação.

Pereira, Costa, Borba e Jurberg (2010, p.395), identificaram numa revisão da produção acadêmica nacional na área de saúde no período entre 1989 a 2009 sobre a construção do conhecimento em Biossegurança 126 artigos publicados em periódicos nacionais na área de saúde, onde destes, 46 tratavam sobre à temática educação, e apenas oito abordaram a prática educacional em biossegurança. Partindo disto, é perceptível a necessidade de estudos mais abrangentes sobre o tema, tendo em vista o ensino e aprendizado em biossegurança nas universidades, principalmente nos cursos da área da saúde. Todavia com a 
repercussão da pandemia e a elevada produção cientifica no período, acredita-se que para os próximos anos haverá um grande volume de artigos que abordem as práticas educacionais em biossegurança durante e pós-pandemia.

Sousa Neto, Bortoluzzi e Freitas (2020, p.1), relataram a existência de uma série de equipamentos que devem ser adotados por profissionais de saúde, sendo que alguns deles possuem recomendações específicas para que sejam reutilizados, dada sua escassez no mercado, devido a busca desenfreada durante a pandemia. $\mathrm{O}$ autor evidenciou também a necessidade dos profissionais da saúde se manterem atualizados e trabalharem conforme as recomendações nacionais e mundiais de saúde, o que remete a responsabilidade para com a saúde do trabalhador e com a sociedade de modo geral.

É imperativo que haja o desenvolvimento de pesquisas na área de Biossegurança e a disseminação do conhecimento destas, partindo do pressuposto que os hábitos e comportamentos humanos deverão se adequar às regras e normas instituídas pelos órgãos sanitários a fim da preservação da vida humana.

\subsection{Biossegurança em Odontologia}

Petrescu, Lucaciu e Roman (2020, p.2), notificaram que os cirurgiões-dentistas são amplamente expostos a serem infectados, porém também podem ser os primeiros a identificar pacientes com SARS-CoV-2 positivo, através do diagnóstico de manifestações orais do COVID-19, como: xerostomia, dor orofacial, úlceras, bolhas, periodontite necrosante, e alterações quimiossensoriais que podem desencadear distúrbios gustativos, como a ageusia e a hipogeusia, sendo estas manifestações secundárias à anosmia (Freitas et al., 2020, p.166). Todavia tais manifestações em pacientes suspeitos e/ou confirmados ainda são variados e inconclusivos, mas os especialistas devem se atentar aos sintomas iniciais a fim de minimizar os impactos da infecção. É válido ressaltar também a importância do dentista como parte da equipe multidisciplinar no apoio a estes pacientes, principalmente aqueles em estado crítico em UTI.

Já Sousa Neto et Al. (2020, p.2), discutiu as indicações relacionadas ao uso dos Equipamentos de Proteção Individual - EPI para profissionais que atuam na assistência aos pacientes suspeitos ou confirmados para a COVID-19, a fim de reduzir ou impedir a elevação no número de casos nestes profissionais. Declarou também que as máscaras de proteção respiratória (N95, N99, N100, PFF2 ou PFF3) não possuem evidências de maior proteção relacionada a prevenção de infecções respiratórias virais quando comparadas com as máscaras médicas, todavia, mediante a situação de pandemia, tiveram indicação exclusiva para os profissionais da saúde que estão atuando na assistência aos pacientes ou realizando procedimentos que geram aerossóis, como é o caso da clínica odontológica, tendo em vista que a transmissão e disseminação do vírus pode ser dada através de gotículas e/ou saliva.

Armond et al. (2016, p. 32), realizou uma pesquisa com servidores, discentes e empregados da limpeza responsáveis pelas clínicas e laboratórios do curso de Odontologia de uma universidade e concluiu que a abordagem pedagógica sobre biossegurança no curso deve ser revisada para que capacite melhor os alunos e assim a distância entre o conhecimento teórico e o prático seja diminuída. Ressaltou também a necessidade de investimento em capacitação de todo o corpo profissional. E isto, evidencia a importância da educação continuada em biossegurança na produção de profissionais qualificados e atualizados.

Quanto ao comportamento dos alunos de Odontologia em relação ao controle de infecção cruzada, Pimentel, Batista Filho, dos Santos e da Rosa (2012, p.525), relataram em análise estatística que alunos dos períodos mais avançados apresentaram melhor comportamento na conduta de organização do instrumental para esterilização e desinfecção de superfícies. 


\section{Biossegurança em procedimentos cirúrgico-odontológicos frente a pandemia}

\subsection{Fatores de riscos}

Tendo em vista o grande potencial de infecção pelo SARS-CoV-2 através da saliva (Xu et al; Li et al.; 2020, p.1), Franco, de Camargo, Peres e de Melo (2020, p.19 e 21) notificaram que o discernimento na prática odontológica é imprescindível e assim sugeriu que os atendimentos neste período pandêmico se limitassem apenas às urgências e emergências. Relataram também a importância da instituição dos cuidados de biossegurança e da adequada paramentação dos cirurgiõesdentistas, que por muito negligenciaram tais medidas, as quais possibilitam menor formação de aerossóis e o exercício seguro da profissão, garantindo assim a saúde do trabalhador.

Ge, Yang, Xia, Fu e Zhang (2020, p.6), relataram a necessidade de compreensão da transmissão de aerossóis e o que estes podem implicar na Odontologia, bem como Franco et al. (2020, p.18), informou que os conhecimentos sobre infecção cruzada, infecções respiratórias e biossegurança devem ser integrados à rotina laboral. E deste modo, tornar possível a identificação e correção das negligências que são corriqueiras na rotina laboral.

Moura, Moura, Pereira e Marinho (2020, p.7283), reiteram que no cenário de pandemia os protocolos que assegurem a segurança no exercício laboral não devem ser negligenciados, alerta que os cirurgiões-dentistas devem manter a frequência na higienização das mãos, estar atentos à desinfecção do ambiente após cada atendimento, do mesmo modo que orienta ao uso correto dos EPI's visando a redução da infecção cruzada entre a equipe e o paciente.

Os fatores de riscos colocam o cirurgião bucomaxilofacial como um dos profissionais mais expostos a contaminação, considerando sua atuação no trauma facial e no tratamento de afecções especificas da categoria, como as infecções odontogênicas (Maia, Dias, Beserra, Cardoso, \& Moreira, 2020, p.3). Guo, Zhou, Liu e Tan (2020, p.1), relataram que as queixas dentárias apresentaram uma mudança significativa com o início da pandemia, onde foi constatado uma redução de $38 \%$ nas entradas em prontos-socorros e uma mudança significativa na distribuição dos problemas dentários, onde casos de infecções dentárias e orais aumentaram cerca de $20 \%$, traumatismos dentários reduziram $4 \%$, ao passo que os casos não urgentes reduziram 30\% com relação aos índices pré-pandêmicos.

\subsection{Recomendações}

Em consonância a Associação Americana de Odontologia (ADA-EUA), o Centro para o Controle e Prevenção de Doenças (CDC- EUA) e o Serviço Nacional de Saúde (NHS/Inglaterra) que avaliaram os riscos da atividade laboral dos cirurgiões-dentistas, com o objetivo de estabelecer medidas de segurança à saúde do trabalhador e dos pacientes (ANVISA, 2020, p.81), o Conselho Federal de Odontologia (CFO) regulamentou o exercício da Odontologia à distância através da Resolução CFO 226/2020.

Tendo em consideração o cenário atual, Meza-palma e Rosales-salas (2020, p.529) desenvolveram a Categorização Remota de Emergência e Assistência Odontológica (CRUDA), devido a inexistência de um protocolo específico de teleodontologia aplicado à triagem de emergência. O método demonstrou viabilidade de aplicação, pois além de possibilitar um atendimento personalizado, permite a otimização do processo de triagem a distância, tornando possível também a redução de riscos da exposição ao SARS-CoV-2. Todavia, ainda se faz necessário mais estudos para a comprovação da efetividade e validade deste instrumento.

Foi regulamentado também que a qualidade e renovação do ar devem ser asseguradas de forma a estabelecer ambientes em que haja segurança, e levando em consideração as formas de transmissão da doença é recomendado o uso de sistema de climatização com exaustão e/ou a manutenção das janelas abertas, para que ocorra a renovação do ar no ambiente clinico-ambulatorial (ANVISA, 2020, p.83). 
Edwards, Kasten, Nelson, Elner e McKean (2020, p.1) informaram que nos Estados Unidos as cirurgias que envolvessem o trato aerodigestivo superior foram canceladas, exceto as que representassem ameaça às funções vitais. Enfatizou ainda quatro pontos de relevância frente ao cenário de pandemia: a garantia da segurança dos pacientes e profissionais de saúde; preservação dos recursos vitais; fornecimento de cuidados essenciais com alta qualidade; e manejo ambulatorial eficiente, quando existir possibilidade. Já Allevi et. Al. (2020, p.694) ressaltou a necessidade da manutenção de cirurgias de emergência, oncológicas, de tumores benignos e/ou malignos, e a suspensão das eletivas.

Ao passo que Wang, Moon, Le e Panchal (2020, p.2) sugeriram a redução no número de membros das equipes cirúrgicas, dar prioridade à realização de tratamentos conservadores, realizar antissepsia no trans-cirúrgico quando houver manejo de mucosa, e que os acessos a cavidade oral e nasal, osteotomias e o uso do cautério sejam evitados.

Destarte é perceptível a existência e possibilidade do exercício seguro da profissão através da execução das medidas que resguardam a vida humana, no entanto a adaptação ao novo pode demandar tempo tanto aos aspectos ergonômicos, quanto econômicos, tendo em vista a elevação dos valores dos equipamentos de proteção individual no mercado.

\subsection{Equipamentos de proteção individual na prática bucomaxilofacial}

Xavier et. al. (2020, p.4493) informou que o atrito promovido pela broca odontológica em alta rotação associado a água nos dentes pode gerar aerossóis, que quando combinados aos fluidos da cavidade bucal geram bioaerossóis. No que é pertinente ao SARS-CoV-2 alertou que o vírus pode permanecer no ar por até oito horas, e partindo deste pressuposto, sugeriuse o refinamento das estratégias de prevenção a fim de evitar tais infecções, através da concentração na higienização das mãos, uso de protetores faciais, máscaras N95 e PFF 2/3, descontaminação com álcool a 70\%, atenção redobrada na esterilização, desinfecção do ambiente, tricotomia da face de pacientes do sexo masculino e evitar procedimentos que não sejam de caráter emergencial ou de urgência.

Edwards (2020, p.1), Careddu (2020 p.17), Panesar (2020, p.1140) também alertaram sobre a importância do uso completo dos EPI, composto por: máscara cirúrgica. PFF2/N-95, protetores faciais, óculos de proteção e capote descartável. Siles-Garcia, Alzamora-Cepeda, Atoche-Socola, Peña-Soto e Arriola-Guillén (2020, p.1) consideraram que as medidas de biossegurança devem contemplar toda a logística do atendimento, compreendendo o antes, durante e o pós, o que torna indispensável a adoção de todas as medidas preventivas, bem como a triagem telefônica, auferição da temperatura e organização da sala de espera.

De acordo com Maia et al. (2020, pp. 7-8) além dos EPI supracitados, se faz necessário o uso do capuz para cabeça e pescoço, e ressalta que a adoção de tais medidas é recomendada pelas entidades que representam a Cirurgia e Traumatologia Bucomaxilofacial, como: o Colégio Brasileiro de Cirurgia e Traumatologia Buco-Maxilo-Faciais (CBCTBMF), American Association of Oral and Maxillofacial Surgeons (AAOMS), French Society of Stomatology, Maxillo-Facial Surgery and Oral Surgery (SFSCMFCO) e AO Foundation Craniomaxilfacial - AOCMF.

Sousa Neto et. Al. (2020, p.2 e 3) salientou no que é pertinente ao uso dos óculos de proteção e protetores faciais frente aos riscos da exposição às secreções corporais, a necessidade de se verificar a integridade destes dispositivos antes de utiliza-los, o que retrata a importância da desinfecção destes materiais que também podem transmitir o vírus de modo cruzado.

A ANVISA (2020, p.83) destacou que as máscaras N95/PFF2 ou equivalentes que contenham válvula expiratória não são apropriadas à prática odontológica, em razão de permitirem a saída do ar expirado pelo profissional, tornando-se um fator de risco. No entanto, caso sejam as únicas disponíveis, devido a demanda do mercado, é recomendado o uso conjunto de protetores faciais, com intuito de amenizar os pontos negativos destes modelos. Todavia, tal medida não é recomendada para a realização de procedimentos cirúrgicos, pois potencializam os riscos de infecção do ambiente. 
Quanto à sequência de paramentação e desparamentação a ANVISA orienta através da NOTA TÉCNICA GVIMS/GGTES/ANVISA No 04/2020 o que é configurado no Quadro 1, porém é válido ressaltar a existência de outros métodos que também podem ser aceitos.

Quadro 1. Sequência de paramentação e desparamentação.

\begin{tabular}{|c|c|}
\hline PARAMENTAÇÃO & DESPARAMENTAÇÃO \\
\hline Higienizar as mãos & Retirar as Luvas \\
\hline Colocar o Avental & Higienizar as mãos \\
\hline Colocar a Máscara N95/PFF2* & Retirar o Protetor Facial \\
\hline Colocar Gorro & Retirar o Óculos \\
\hline Colocar o Óculos & Retirar o Gorro \\
\hline Colocar o Protetor Facial & Higienizar as mãos \\
\hline Higienizar as mãos & Retirar a Máscara N95/PFF2 \\
\hline Colocar as Luvas & Higienizar as mãos. \\
\hline - & \\
\hline
\end{tabular}

Fonte: ANVISA (2020).

Partindo do que é constatado no quadro acima é perceptível a necessidade de cautela no manejo dos equipamentos de proteção individual na prática odontológica, principalmente no ato de desparamentação, onde a higienização das mãos é enfatizada, a fim de minimizar as chances de infecção pelo SARS-CoV-2 para o profissional.

\section{Conclusões}

É imprescindível que os cirurgiões-dentistas se adequem às medidas de biossegurança recomendadas pelas instituições e órgãos que regulamentam a vigilância em saúde a fim de que os procedimentos cirúrgicos odontológicos possam ser executados com segurança. E partindo disto, propiciar o exercício pleno da Odontologia em detrimento ao impacto da infecção pelo SARS-CoV-2 na vida humana.

Os cirurgiões bucomaxilofaciais devem seguir as normas de paramentação e desparamentação e uso apropriado de equipamentos de proteção individual, higienização, desinfecção e esterilização rigorosamente tendo em vista a grande exposição aos bioaerossóis. É válido evidenciar também a necessidade de novos protocolos de triagem emergencial à distância ou o aprimoramento dos já existentes, considerando os benefícios que estes possibilitam e o papel que podem desempenhar não somente na prática cirúrgica em contexto de pandemia, mas na Odontologia de modo geral.

Todavia é necessário enfatizar a necessidade de estudos que abordem a fiscalização da prática odontológica em contexto de pandemia, assim como o impacto da elevação dos valores dos equipamentos de proteção individual nas clínicas de pequeno porte.

\section{Referências}

Agência Nacional de Vigilância Sanitária. Nota técnica GVIMS/GGTES/ANVISA n. 04/2020: orientações para serviços de saúde: medidas de prevenção e controle que devem ser adotadas durante a assistência aos casos suspeitos ou confirmados de infecção pelo novo coronavírus (SARS-CoV-2). Brasília, DF: 
Research, Society and Development, v. 10, n. 1, e5810111401, 2021

(CC BY 4.0) | ISSN 2525-3409 | DOI: http://dx.doi.org/10.33448/rsd-v10i1.11401

ANVISA; 2020. $\quad$ http://portal.anvisa.gov.br/documents/33852/271858/Nota+T\%C3\%A9cnica+n+04-2020+GVIMS-GGTES-ANVISA-

ATUALIZADA/ab598660-3de4-4f14-8e6f-b9341c196b28.

Allevi, F., Dionisio, A., Baciliero, U., Balercia, P., Beltramini, G. A., Bertossi, D., Bozzetti, A., Califano, L., Cascone, P., Colombo, L., Copelli, C., De Ponte, F. S., De Riu, G., Della Monaca, M., Fusetti, S., Galié, M., Giannì, A. B., Longo, F., Mannucci, N., Nocini, P. F., \& Biglioli, F. (2020). Impact of COVID-19 epidemic on maxillofacial surgery in Italy. The British journal of oral \& maxillofacial surgery, 58(6), 692-697. https://doi.org/10.1016/j.bjoms.2020.04.035

Armond, A. C. V., Gonçalves, P. F., Flecha, O. D., Oliveira, D. W. D., de Sampaio, F. C., Falci, S. G. M. Conhecimentos de biossegurança para as principais atividades de risco envolvendo serviços públicos, discentes e empregados da limpeza do curso de odontologia da UFVJM/Dia mantina. RBOL. 2016; 3(2), 3252 .

Careddu, R., Ciaschetti, M., Creavin, G., Molina, F., \& Plotino, G. (2020). COVID-19 and dental practice: overview and protocols during pandemic. Giornale Italiano di Endodonzia, 34(1).

Edwards, S. P., Kasten, S., Nelson, C., Elner, V., \& McKean, E. (2020). Maxillofacial trauma management during COVID-19: multidisciplinary recommendations. Facial Plastic Surgery \& Aesthetic Medicine, 22(3), 157-159.

Freitas, J.A., Cavalcante, L.S., \& Lopes, I.A. (2020). Manifestações orais do covid-19: o impacto do vírus na saúde bucal. XVIII Jornada Acadêmica da UFPI. Teresina - PI. Disponivel em: https://drive.google.com/file/d/1dZNAZ2oMfGcuYSlse0pXi3bQ4_NGL-Xw/view

Ge, Z. Y., Yang, L. M., Xia, J. J., Fu, X. H., \& Zhang, Y. Z. (2020). Possible aerosol transmission of COVID-19 and special precautions in dentistry. Journal of Zhejiang University-Science $\mathrm{B}, 1-8$.

Guo, H., Zhou, Y., Liu, X., \& Tan, J. (2020). The impact of the COVID-19 epidemic on the utilization of emergency dental services. Journal of dental sciences, $10.1016 /$ j.jds.2020.02.002. Advance online publication. doi.org/10.1016/j.jds.2020.02.002

JB, F., De Camargo, A. R., \& MPSM, P. (2020). Cuidados Odontológicos na era do COVID-19: recomendações para procedimentos odontológicos e profissionais. Rev Assoc Paul Cir Dent, 74(1), 18-21.

Li, Y., Zhou, W., Yang, L., \& You, R. (2020). Physiological and pathological regulation of ACE2, the SARS-CoV-2 receptor. Pharmacological research, 157, 104833. doi.org/10.1016/j.phrs.2020.104833.

Ludwig, S., \& Zarbock, A. (2020). Coronaviruses and SARS-CoV-2: A Brief Overview. Anesthesia and analgesia, 131(1), 93-96. doi.org/10.1213/ANE.0000000000004845.

Maia, S. E. S., Dias, L. P. S., Beserra, P. S., Cardoso. L. I. S., Silva, K. R. V., Moreira, T. C. A. (2020). Recomendações de biossegurança para a atuação do cirurgião bucomaxilofacial na assistência a pacientes no período de pandemia pelo Coronavírus. JCS HU-UFPI. Ed. Espec. 3(Supl.2):e-11208 ISSN: 25950290 Recuperado de https://revistas.ufpi.br/index.php/rehu/article/viewFile/11208/pdf.

Marcele, J. P., Mário M. V. B. F., Jozemar, P. dos S., Marize, R. D.R.(2012). Scielo, Biossegurança: comportamento dos alunos em relação ao controle de infecção cruzada. Universidade Federal da Paraíba (UFPB), João Pessoa (PB), Brasil.

Meza-P. L., \& Rosales-S. J. (2020). Protocolo de teleodontología para asistencia al paciente en el manejo de urgencia dental. Cuarentena COVID-19 (SARSCoV-2). Categorización remota de urgencia dental y asistenci (C.R.U.D.A.). Int. J. Odontostomat., 14(4):529-537, doi.org/10.4067/S0718$381 \times 2020000400529$

Moura, J., Moura, K., Pereira, R., \& Marinho, R. (2020). COVID-19: A odontologia frente à pandemia. Brazilian Journal of Health Review. 3. 7276-7285. 10.34119/bjhrv3n4-006.

Panesar, K., Dodson, T., Lynch, J., Bryson-Cahn, C., Chew, L., \& Dillon, J. (2020). Evolution of COVID-19 Guidelines for University of Washington Oral and Maxillofacial Surgery Patient Care. Journal of Oral and Maxillofacial Surgery. 
Research, Society and Development, v. 10, n. 1, e5810111401, 2021

(CC BY 4.0) | ISSN 2525-3409 | DOI: http://dx.doi.org/10.33448/rsd-v10i1.11401

Pereira, M. E. C., Costa, M. A. F. B., Cintia, M., \& Jurberg, C. (2010). Construção do conhecimento em biossegurança: uma revisão da produção acadêmica nacional na área de saúde (1989-2009). Saúde e Sociedade, 19(2), 395-404. doi.org/10.1590/S0104-12902010000200015.

Petrescu, N., Lucaciu, O., Roman, A. (2020). Oral mucosa lesions in COVID-19 [published online ahead of print, Jun 19]. Oral Dis.10.1111/ odi.13499. doi:10.1111/ odi.13499.

RESOLUÇÃO CFO-226, de 04 de junho de 2020. Dispõe sobre o exercício da Odontologia a distância, mediado por tecnologias, e dá outras providências. http://sistemas.cfo.org.br/visualizar/atos/RESOLU\%C3\%87\%C3\%83O/SEC/2020/226

Neto, R. S. A., Batista, B. B., \& Reis, J. de F. D. (2020). Equipamentos de proteção individual para prevenção de infecção por Sars-Cov-2. JMPHC | Journal of Management \& Primary Health Care | ISSN 2179-6750, 12, 1-7. doi.org/10.14295/jmphc.v12.985.

Segata, J. (2020). Covid-19, biossegurança e antropologia. Horizontes Antropológicos, 26(57), 275-313. doi.org/10.1590/s0104-71832020000200010.

Siles, G. A. A., Alzamora, C. A. G., Atoche, S. K. J., Peña, S, C., \& Arriola, G. L. E. (2020). Biosafety for Dental Patients During Dentistry Care After COVID-19: A Review of the Literature. Disaster medicine and public health preparedness, 1-6. doi.org/10.1017/dmp.2020.252.

Teixeira, P., \& Valle, S. (2010). Biossegurança: uma abordagem multidisciplinar. (2a ed.), Rio de Janeiro, RJ: Fiocruz, 442p.

Xavier, T. B. et al. (2020). Protocolo de Tratamento Odontológico na Cirurgia e Traumatologia Buco-Maxilo-Facial no Contexto do COVID-19/Dental Treatment Protocol in Buco-Maxillofacial Surgery in the Context of COVID-19. Brazilian Journal of Health Review, 3(3), 4484-4500.

Xu, H., Zhong, L., Deng, J., Peng, J., Dan, H., Zeng, \& Chen, Q. (2020). High expression of ACE2 receptor of 2019-nCoV on the epithelial cells of oral mucosa. International journal of oral science, 12(1), 8. doi.org/10.1038/s41368-020-0074-x.

Wang, T. T., Moon, H.S., Le, A., Panchal, N. (2020). Proceedings of the OMS COVID-19 Response Conference [published online ahead of print. May 11]. J Oral Maxillofac Surg. 2020;78(8),1268-1274. 